\title{
Comparison of 5-Year Outcomes of Robot-Assisted Laparoscopic and Laparoscopic Partial Nephrectomy in Patients With Localized Renal Cell Carcinoma
}

\author{
Chung Un Lee ${ }^{1}$, Minyong Kang ${ }^{1}$, Hyun Hwan Sung ${ }^{1}$, Hwang Gyun Jeon ${ }^{1}$, \\ Deok Hyun Han', Byung Chang Jeong', Seong Soo Jeon', Hyun Moo Lee', \\ Han Yong Choi ${ }^{2}$, Seong II Seo'
}

\footnotetext{
${ }^{1}$ Department of Urology, Samsung Medical Center, Sungkyunkwan University School of Medicine, Seoul, Korea
}

${ }^{2}$ Department of Urology, Kangbuk Samsung Hospital, Sungkyunkwan University School of Medicine, Seoul, Korea

\begin{abstract}
Purpose: To compare the 5-year oncologic and functional outcomes of robot-assisted laparoscopic partial nephrectomy (RALPN) and laparoscopic partial nephrectomy (LPN) as treatment for localized renal cell carcinoma (RCC).

Materials and Methods: We analyzed the records of 181 patients with localized RCC who underwent RALPN $(n=97)$ or LPN ( $n=84)$ between 2007 and 2011. Demographic and preoperative data with estimated glomerular filtration rate (eGFR), intraoperative data including warm ischemic time (WIT) and complications, oncologic outcomes (recurrence, metastasis), and rate of eGFR preservation at most recent follow-up were examined. Results: WIT was shorter in the RALPN group $(27 \pm 9.1$ minutes) than the LPN group $(31 \pm 10$ minutes, $p=0.019)$. Intraoperative complication rates were also lower in RALPN patients than LPN patients $(4.1 \%$ vs. $14.3 \%)$. The eGFR preservation rate was higher in the RALPN group (84.6\%) than in the LPN group (81.5\%, $p=0.049$ ). Particularly, a relatively high difference in the eGFR preservation rate was observed in the RALPN group compared with the LPN group according to R.E.N.A.L. score $7-10$ values (RALPN, $86.5 \pm 12.9$ vs. LPN, $76.7 \pm 16.0 ; p=0.003$ ). During the follow-up period, there was no local recurrence in either group and distant metastases only occurred in one patient in the RALPN group and in 2 patients in the LPN group.

Conclusions: RALPN and LPN showed similar 5-year oncologic outcomes, but RALPN was superior to LPN in terms of WIT, intraoperative complications, and long-term eGFR preservation rate, especially in complex cases. (Korean J Urol Oncol 2017;15:172-177)
\end{abstract}

Key Words: Renal cell carcinoma $\cdot$ Robot-assisted laparoscopic partial nephrectomy $\cdot$ Laparoscopic partial nephrectomy $\cdot$ Oncologic outcomes $\cdot$ Functional outcomes

Received November 3, 2017, Revised November 8, 2017,

Accepted November 20, 2017

Corresponding Author: Seong Il Seo

Department of Urology, Samsung Medical Center, Sungkyunkwan University School of Medicine, 81 Irwon-ro, Gangnam-gu, Seoul 06351, Korea

E-mail: seongil.seo@samsung.com

Tel: +82-2-3410-3556, Fax: +82-2-3410-6992

ORCID code: https://orcid.org/0000-0002-9792-7798

\section{INTRODUCTION}

There is a recent increase in the development of imaging techniques and health screening systems for detection of small renal masses (SRM). Surgery remains the main treatment option for SRM and partial nephrectomy is currently the standard treatment for SRM, replacing the formerly common nephron-sparing surgery (NSS). ${ }^{1}$

The decrease in the number of conventional open partial

(c) (i) (5) This is an Open Access article distributed under the terms of the Creative Commons Attribution Non-Commercial License (http://creativecommons.org/licenses/by-nc/4.0/) which permits unrestricted non-commercial use, distribution, and reproduction in any medium, provided the original work is properly cited. 2017 (C) Copyright The Korean Urological Oncology Society and The Korean Prostate Society. All Rights Reserved. 
nephrectomies (OPNs) performed is due to an increase in the number of laparoscopic partial nephrectomies (LPNs), which is a minimally invasive surgery. However, patients' physical characteristics, such as the size, position, and structure of the kidneys, as well as the physician's experience level, can limit the efficacy of LPN.

Recently, robot-assisted LPN (RALPN) has replaced LPN because it allows for more ergonomic movement and offers easier suturing technologies. ${ }^{2,3}$ Also, it is widely known that RALPN yields better surgical outcomes than LPN. ${ }^{4,5}$

There are a number of reports on the oncological and functional outcomes during 3 years of observation after RPN, but there are few reports on the long-term outcomes (e.g., $>5$ years) associated with RPN. ${ }^{6-8}$ Furthermore, there are even fewer comparative studies of the oncological and functional outcomes from RPN and LPN after 5 years of observation.

Herein, we aimed to compare the oncological and functional outcomes of RALPN and LPN over 5 years of postsurgery follow-up.

\section{MATERIALS AND METHODS}

We retrospectively reviewed clinical data from 112 renal cell cancer (RCC) patients who underwent RALPN and 95 patients who underwent LPN at Samsung Medical Center by single surgeon (S.I.S) from October 2007 to December 2012. After excluding 26 patients for having bilateral RCC or incomplete clinical data, a total of 181 patients were finally included in this study.

Age at the time of surgery, sex, body mass index, preoperative estimated glomerular filtration rate (eGFR), tumor size, American Society of Anesthesiologists physical status classification grade, R.E.N.A.L. nephrometry score, ${ }^{9}$ and diethylene tetramine penta-acetic acid (DTPA) kidney scan for measuring differential renal function were included as demographic data and preoperative variables.

We calculated eGFR using the Modification of Diet Renal Disease Study Group formula. ${ }^{10}$ Tumor size was measured using the largest tumor diameter following the axis on the computed tomography (CT) scan, and tumor complexity was determined by the R.E.N.A.L. score. Renal volume was measured by 3D support system (Xelis, Infinitt, Seoul, Korea) and renal volume preservation rate was presented as percentage by dividing the recently measured volume of the kidney by the volume of the preoperative kidney.

Estimated blood loss (EBL), warm ischemia time (WIT), and length of hospital stay were included as perioperative and postoperative variables. Complications after surgery were classified using the Clavien-Dindo classification system. ${ }^{11}$

Tumor tissue pathology was based on the 2004 World Health Organization criteria; ${ }^{12}$ nuclear grade classification followed the Fuhrman scheme; and clinical stage were based on the 2010 American Joint Committee of Cancer Staging Manual, seventh edition. ${ }^{13}$ The surgeon selected the route of surgical approach (e.g., transperitoneal, retroperitoneal) according to tumor position, size, and structure.

We followed-up with patients 1, 3, 6, and 12 months after surgery, and then annually thereafter. Evaluation after surgery was conducted through routine laboratory tests at an outpatient department. e-GFR was recorded as part of routine laboratory tests at every follow-up visit and DTPA scans were taken 3 or 6 months and 12 months postsurgery and, if necessary, annually thereafter. Recurrence and metastasis were evaluated via CT scanning 6 months and 12 months after surgery, and annually thereafter.

This study was approved by the Institutional Review Board of Samsung Medical Center (approval number: 2017-10-117).

To compare clinicopathological characteristics of the 2 groups, Mann-Whitney U-tests were conducted for continuous variables and chi-square tests was conducted for categorical variables. Data are reported as means and standard deviations or medians and interquartile range (IQR) for continuous variables and as absolute values and percentages for categorical variables. Kaplan-Meier survival analysis was used to estimate disease-free survival and the log-rank test was used to determine the statistical significance of survival differences between the 2 groups. Values of $\mathrm{p}<0.05$ were considered statistically significant. IBM SPSS Statistics ver. 20.0 (IBM Co., Armonk, NY, USA) was used for all statistical analyses.

\section{RESULTS}

We analyzed 97 patients who underwent RALPN and 84 patients underwent LPN to treat localized RCC. Table 1 shows the baseline clinicopathological characteristics of the patients. Notably, there were no significant differences in baseline characteristics between the 2 groups.

As shown in Table 2, there were no significant differences 
Table 1. Demographic and preoperative data for patients undergoing robotic-assisted laparoscopic partial nephrectomy (RALPN) or laparoscopic partial nephrectomy (LPN)

\begin{tabular}{lccc}
\hline \multicolumn{1}{c}{ Variable } & $\begin{array}{c}\text { RALPN } \\
(\mathrm{n}=97)\end{array}$ & $\begin{array}{c}\text { LPN } \\
(\mathrm{n}=84)\end{array}$ & p-value \\
\hline Sex & & & \\
$\quad$ Male & $69(71.1)$ & $52(61.9)$ & 0.188 \\
$\quad$ Female & $28(28.9)$ & $32(38.1)$ & \\
Age (yr) & $51.5 \pm 11.4$ & $53.3 \pm 11.9$ & 0.298 \\
Body mass index $\left(\mathrm{kg} / \mathrm{m}^{2}\right)$ & $24.6 \pm 3.0$ & $25.2 \pm 3.6$ & 0.217 \\
Preoperative eGFR & $88.6 \pm 15.6$ & $87.6 \pm 19.9$ & 0.810 \\
Tumor size (cm) & $2.7 \pm 0.9$ & $2.4 \pm 1.1$ & 0.140 \\
ASA PS classification grade & $1.5 \pm 0.5$ & $1.5 \pm 0.6$ & 0.367 \\
R.E.N.A.L. score & $6(5-8)$ & $6(5-8)$ & 0.779 \\
$\quad$ 4-6 & $54(55.7)$ & $47(56.0)$ & 0.970 \\
$\quad 7-10$ & $43(44.3)$ & $37(44.0)$ & \\
Clinical stage & & & \\
$\quad$ T1a & $86(88.7)$ & $79(94.0)$ & 0.203 \\
T1b & $11(11.3)$ & $5(6.0)$ & \\
\hline
\end{tabular}

Values are presented as number (\%), mean \pm standard deviation, or median (interquartile range).

e-GFR: estimated glomerular filtration rate, ASA PS: American Society of Anesthesiologists physical status. in EBL, length of hospital stay, or positive surgical margin rates between the 2 groups. Interestingly, while the mean operative time was longer in the RALPN group ( $270 \pm 82$ minutes) than in the LPN group $(200 \pm 72$ minutes, $\mathrm{p}<0.001)$, WIT was significantly shorter in patients who underwent RALPN than in those who underwent LPN (RALPN, $27 \pm 9.1$ minutes vs. LPN, $31 \pm 10$ minutes, $p=0.019$ ). Furthermore, patients who underwent RALPN exhibited fewer intraoperative complications than patients who underwent LPN (RALPN, $4.1 \%$ vs. LPN, $14.3 \%$, $\mathrm{p}=0.016$ ). However, there were no differences in the postoperative complication rates between the 2 groups.

The median follow-up duration was 60.2 months (IQR, 52.955.7 months) for RALPN patients and 63.5 months (IQR, 54.976.4 months) for LPN patients. During the follow-up time, there were no local recurrences in either patient group and only 3 cases developed distant metastases, 1 in the RALPN group and 2 in the LPN group.

Additionally, the 5-year cancer-free survival (CFS) rate was

Table 2. Perioperative, postoperative, and pathologic data

\begin{tabular}{|c|c|c|c|}
\hline Variable & RALPN & LPN & $\mathrm{p}^{\text {-value }}$ \\
\hline \multicolumn{4}{|l|}{ Intraoperative outcomes } \\
\hline Operation time (min) & $240 \pm 82$ & $200 \pm 72$ & $<0.001$ \\
\hline WIT (min) & $27 \pm 9.1$ & $31 \pm 10$ & 0.019 \\
\hline EBL (mL) & $216 \pm 169$ & $195 \pm 173$ & 0.417 \\
\hline Intraoperative complications (Clavien-Dindo classification grade $\geq$ II) & $4(4.1)$ & $12(14.3)$ & \\
\hline Blood transfusion & $1(1.0)$ & $1(1.2)$ & 0.016 \\
\hline Ureteric injury & $0(0)$ & $1(1.2)$ & \\
\hline Adjacent organ injury & $1(1.0)$ & $3(3.6)$ & \\
\hline Conversion to $\mathrm{RN}$ & $1(1.0)$ & $3(3.6)$ & \\
\hline Conversion to HALS-PN & $1(1.0)$ & $4(4.8)$ & \\
\hline Postoperative complications (Clavien-Dindo classification grade) & $7(7.2)$ & $5(6.0)$ & \\
\hline II & $7(7.2)$ & $1(1.2)$ & 0.733 \\
\hline III & $0(0)$ & $4(4.8)$ & \\
\hline LOS (day) & $7(7-7)$ & $7(7-8)$ & 0.826 \\
\hline Positive surgical margin & $0(0)$ & $1(1.2)$ & 0.464 \\
\hline Follow-up (mo) & $60.2(52.9-66.7)$ & $63.5(54.9-76.4)$ & 0.127 \\
\hline \multicolumn{4}{|l|}{ Histopathology } \\
\hline Clear cell RCC & $78(80.4)$ & $73(86.9)$ & 0.271 \\
\hline Papillary RCC & $9(9.3)$ & $4(4.8)$ & \\
\hline Chromophobe RCC & $9(9.3)$ & $4(4.8)$ & \\
\hline Others & $1(1.0)$ & $3(3.6)$ & \\
\hline Recurrence & $0(0)$ & $0(0)$ & \\
\hline Metastasis & $1(1.0)$ & $2(2.4)$ & 0.598 \\
\hline
\end{tabular}

Values are presented as mean \pm standard deviation, number (\%), or median (interquartile range).

RALPN: robotic-assisted laparoscopic partial nephrectomy, LPN: laparoscopic partial nephrectomy, WIT: warm ischemic time, EBL: estimated blood loss, RN: radical nephrectomy, HALS-PN: hand-assisted laparoscopic partial nephrectomy, LOS: length of stay, RCC: renal-cell carcinoma. 
98.9\% (95\% confidence interval [CI], 96.9\%-99.9\%) among RALPN patients and $98.7 \%$ (95\% CI, 96.2\%-99.2\%) among LPN patients (Fig. 1); these rates were not statistically different.

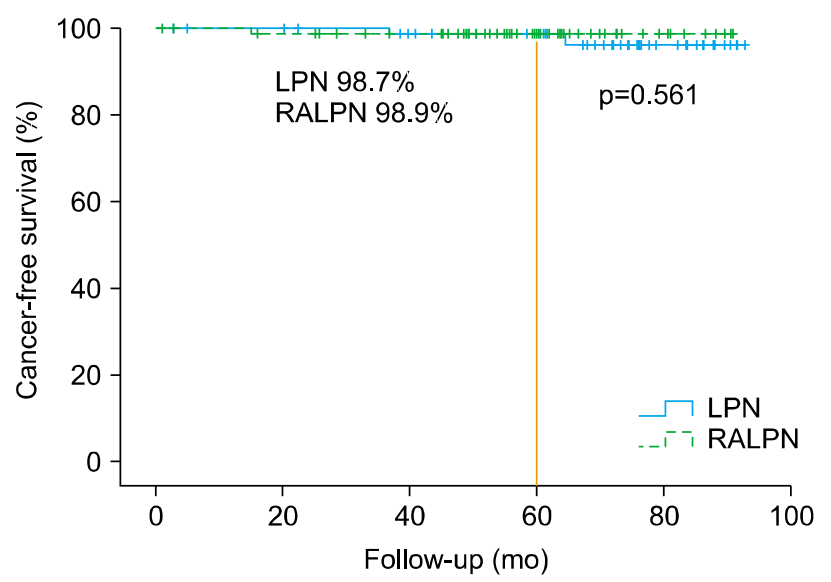

Fig. 1. Kaplan-Meier analysis for estimating cancer-free survival for patients underwent robot-assisted laparoscopic partial nephrectomy (RALPN) and laparoscopic partial nephrectomy (LPN), respectively, for treating localized renal cell carcinoma.
Regarding functional outcomes, eGFR preservation rates measured at last follow-up appointment were significantly higher among RALPN than LPN patients (RALPN, $84.6 \%$ vs. LPN, $81.5 \%, \mathrm{p}=0.049$ ). However, there was no difference in percentage volume of preservation among the operated kidneys or in progression to chronic kidney disease III-V 1 year after surgery (Table 3).

When we performed subgroup analyses on the patients for whom we had R.E.N.A.L. scores, we found no statistical differences in intraoperative outcomes, with the exception of operation time among patients with R.E.N.A.L. score values 4-6 (RALPN, $240.6 \pm 82.9$ vs. LPN, 192.0 $\pm 61.7 ; p=0.001$ ). In particular, eGFR preservation rates measured at the last follow-up point among patients with R.E.N.A.L. score values 4-6 (low complexity) were not significantly different from those in patients with R.E.N.A.L. score values 7-10 (moderate to high complexity). eGFR preservation rates measured at the last follow-up point were significantly higher among the RALPN patients than in the LPN patients (RALPN, $86.5 \pm 12.9$ vs. LPN,

Table 3. Postoperative renal functional outcomes

\begin{tabular}{lccc}
\hline \multicolumn{1}{c}{ Variable } & RALPN & LPN & p-value \\
\hline Preoperative eGFR $(\%)$ & $87.1(80-95.5)$ & $89.2(73.8-99.6)$ & 0.810 \\
Volume preservation of operated kidney after 1 year $(\%)$ & $79.2(71.0-85.9)$ & $77.1(71.0-85.8)$ & 0.685 \\
eGFR preservation at most recent follow-up $(\%)$ & $84.6(78.2-95.1)$ & $81.5(73.9-89.4)$ & 0.049 \\
Postoperative progression to CKD III-V & $10(10.3)$ & $14(16.7)$ & 0.208 \\
\hline
\end{tabular}

Values are presented as median (interquartile range) or number (\%).

RALPN: robotic-assisted laparoscopic partial nephrectomy, LPN: laparoscopic partial nephrectomy, eGFR: estimated glomerular filtration rate, CKD: chronic kidney disease.

Table 4. Intraoperative outcomes, postoperative complications, and eGRF preservation in RALPN and LPN patients according to R.E.N.A.L. score

\begin{tabular}{|c|c|c|c|c|c|c|}
\hline \multirow[b]{2}{*}{ Variable } & \multicolumn{3}{|c|}{ R.E.N.A.L. score 4-6 $(n=101)$} & \multicolumn{3}{|c|}{ R.E.N.A.L. score 7-10 $(\mathrm{n}=80)$} \\
\hline & $\begin{array}{c}\text { RALPN } \\
(\mathrm{n}=54)\end{array}$ & LPN $(n=47)$ & p-value & $\begin{array}{c}\text { RALPN } \\
(n=43)\end{array}$ & LPN $(n=37)$ & $\mathrm{p}^{\text {-value }}$ \\
\hline \multicolumn{7}{|l|}{ Intraoperative outcomes } \\
\hline Operation time (min) & $240.6 \pm 82.9$ & $192.0 \pm 61.7$ & 0.001 & $239.6 \pm 82.9$ & $211.9 \pm 83.9$ & 0.143 \\
\hline Warm ischemic time (min) & $26.3 \pm 9.2$ & $29.5 \pm 10.8$ & 0.106 & $29.5 \pm 8.9$ & $33.5 \pm 10.4$ & 0.077 \\
\hline Estimated blood loss (mL) & $213.3 \pm 184.0$ & $190.0 \pm 187.8$ & 0.531 & $220.5 \pm 151.3$ & $203.0 \pm 155.2$ & 0.612 \\
\hline Postoperative complications (Clavien-Dindo classification grade) & $3(5.6)$ & $3(6.4)$ & & $4(9.3)$ & $2(5.4)$ & \\
\hline II & $3(5.6)$ & $1(2.1)$ & & $4(9.3)$ & $0(0)$ & \\
\hline III & $0(0)$ & $2(4.3)$ & & $0(0)$ & $2(5.4)$ & \\
\hline eGFR preservation at most recent follow-up (\%) & $87.2 \pm 14.1$ & $86.9 \pm 17.1$ & 0.906 & $86.5 \pm 12.9$ & $76.7 \pm 16.0$ & 0.003 \\
\hline
\end{tabular}

Values are presented as mean \pm standard deviation or number $(\%)$.

eGFR: estimated glomerular filtration rate, RALPN: robotic-assisted laparoscopic partial nephrectomy, LPN: laparoscopic partial nephrectomy. 
$76.7 \pm 16.0 ; p=0.003)$ (Table 4).

\section{DISCUSSION}

Recently, minimally invasive partial nephrectomy has become the standard surgical treatment for $\mathrm{SRM}^{1}{ }^{1}$ and comparisons between the outcomes from partial nephrectomy and ordinary surgery are receiving a lot of attention. Long-term oncological and functional outcomes from OPN and LPN are well established $^{14-17}$ but the long-term oncological and functional outcomes from RALPN and comparisons between RALPN and LPN have not been reported. This is the first study from South Korea to compare the long-term oncological and functional outcomes of RALPN and LPN.

Most of the patients in this study had stage T1a (RALPN, 86 [88.7\%] vs. LPN, 79 [94.0\%]; p=0.203), and the complexity of their tumors according to their R.E.N.A.L. score was generally low (RALPN, 6 vs. LPN, 6, p=0.779) because this study was performed by a surgeon with relatively little experience with the technique. According to Reddy et al., ${ }^{18}$ the R.E.N.A.L. score allows physicians to predict the incidence of complications and renal function after PN. Accordingly, both groups' scores are considered good.

Although the operation time was longer for RALPN (RALPN, 240 \pm 82 minutes vs. LPN, $200 \pm 72$ minutes, p $<0.001$ ), intraoperative complications occurred more frequently in LPN patients (RALPN, 4 [4.1\%] vs. LPN, 12 [14.3\%], $\mathrm{p}=0.016)$. We assumed that the longer operation time in RALPN is result from time of docking and dedocking of robot system. If the operation time in RALPN was measured by console time only, it would be same or shorter than time of LPN. However, there were no surgery-related life-threatening side effects (Clavien-Dindo classification grade IV, V) in either group. We found that RALPN was more effective than LPN in terms of complication incidence.

According to Khalifeh et al., ${ }^{7}$ RALPN yielded a 3-year overall survival (OS) of 97.0\%, a cancer-specific survival (CSS) of 99.0\%, and a CFS of $97.0 \%$. Andrade et al. ${ }^{8}$ reported an OS of $91.1 \%$, a CSS of $97.8 \%$, and a CFS of $97.8 \%$ after 5 years. We achieved a CFS of $98.9 \%$ after 5 years for the RALPN patients, which demonstrates the oncological effectiveness of RALPN and this outcome is comparable to the CFS of $98.7 \%$ after 5 years for the LPN patients.

Khalifeh et al. ${ }^{7}$ reported a preoperative GFR of $88.2 \pm 0.8$
$\mathrm{mL} / \mathrm{min} / 1.73 \mathrm{~m}^{2}$ and a most-recent postoperative GFR of $80 \pm 24 \mathrm{~mL} / \mathrm{min} / 1.73 \mathrm{~m}^{2}$, with an $8 \% \pm 17.4 \%$ change after RALPN for a 3-year follow-up period. According to Kim et al., ${ }^{19}$ eGFR recovered to $95.2 \%$ of the preoperative level 5 years postoperatively in RALPN patients and $92.6 \%$ in LPN patients. In this study, the percentage of eGFR preservation at final tracking was $84.6 \%$ in RALPN patients and $81.5 \%$ in LPN patients. We confirmed that RALPN yields superior results for eGFR preservation. We further found that WIT is the main factor associated with preserving renal function because WIT in RALPN patients is shorter than in LPN patients (RPN, 27 \pm 9.1 minutes vs. LPN, $31 \pm 10$ minutes; $p=0.019$ ), and volume preservation is not meaningful. This is in accordance with results of a study by Bessede et al. ${ }^{20}$ that showed that time clamping techniques were associated with preservation of renal function in NSS; however, our results also contradict a study by Ginzburg et al. ${ }^{21}$ that found that residual functional parenchymal volume was the main predictor of ultimate renal function after NSS.

Furthermore, we showed that RALPN yielded superior renal function preservation for patients with intermediate to high R.E.N.A.L. scores $(7-10 ;$ RALPN, $86.5 \pm 12.9$ vs. LPN, $76.7 \pm 16.0, \mathrm{p}=0.003)$. These findings may further indicate that RALPN is even more beneficial for complex cases.

Because this is a retrospective study, the value of our findings is limited by possible selection biases and because we could not control or match between the groups. The impact of our results is further limited by the small sample size and because the study was conducted by a single researcher at a single institution. Especially, in case of DTPA, data is not sufficient for statistical analysis due to the substantial amount of missing data. However, our results are based on 5 years of observations of RALPN patients and this is the first study to compare RALPN and LPN.

The lack of local recurrence and death among our patients is probably due to the selection of patients, the sample size, and the observation period, and could be different in a study with more patients and a longer observation period.

\section{CONCLUSIONS}

In summary, RALPN yielded similar oncological and better functional long-term outcomes than LPN. Particularly, eGFR preservation was significantly superior in patients with complex cases who underwent RALPN than those who were treated with 
LPN. Our data suggest that RALPN can be a primary option for surgical treatment of SRM.

\section{CONFLICT OF INTEREST}

The authors claim no conflicts of interest.

\section{REFERENCES}

1. Meeks JJ, Gonzalez CM. Standard of care for small renal masses in the 21st century. JAMA Surg 2015;150:672-3.

2. Stifelman MD, Caruso RP, Nieder AM, Taneja SS. Robot-assisted laparoscopic partial nephrectomy. JSLS 2005;9:83-6.

3. Patel HD, Mullins JK, Pierorazio PM, Jayram G, Cohen JE, Matlaga BR, et al. Trends in renal surgery: robotic technology is associated with increased use of partial nephrectomy. J Urol 2013;189:1229-35.

4. Wu Z, Li M, Song S, Ye H, Yang Q, Liu B, et al. Propensity-score matched analysis comparing robot-assisted with laparoscopic partial nephrectomy. BJU Int 2015;115:437-45.

5. Shiroki R, Fukami N, Fukaya K, Kusaka M, Natsume T, Ichihara $\mathrm{T}$, et al. Robot-assisted partial nephrectomy: superiority over laparoscopic partial nephrectomy. Int J Urol 2016;23:122-31.

6. Kyllo RL, Tanagho YS, Kaouk JH, Stifelman MD, Rogers CG, Hillyer SP, et al. Prospective multi-center study of oncologic outcomes of robot-assisted partial nephrectomy for pT1 renal cell carcinoma. BMC Urol 2012;12:11.

7. Khalifeh A, Autorino R, Eyraud R, Samarasekera D, Laydner $\mathrm{H}$, Panumatrassamee K, et al. Three-year oncologic and renal functional outcomes after robot-assisted partial nephrectomy. Eur Urol 2013;64:744-50.

8. Andrade HS, Zargar H, Caputo PA, Akca O, Kara O, Ramirez $\mathrm{D}$, et al. Five-year oncologic outcomes after transperitoneal robotic partial nephrectomy for renal cell carcinoma. Eur Urol 2016;69:1149-54.

9. Kutikov A, Uzzo RG. The R.E.N.A.L. nephrometry score: a comprehensive standardized system for quantitating renal tumor size, location and depth. J Urol 2009;182:844-53.

10. Levey AS, Bosch JP, Lewis JB, Greene T, Rogers N, Roth D. A more accurate method to estimate glomerular filtration rate from serum creatinine: a new prediction equation. Modification of Diet in Renal Disease Study Group. Ann Intern
Med 1999;130:461-70.

11. Dindo D, Demartines N, Clavien PA. Classification of surgical complications: a new proposal with evaluation in a cohort of 6336 patients and results of a survey. Ann Surg 2004;240: 205-13.

12. Lopez-Beltran A, Scarpelli M, Montironi R, Kirkali Z. 2004 WHO classification of the renal tumors of the adults. Eur Urol 2006;49:798-805.

13. Edge SB, Compton CC. The American Joint Committee on Cancer: the 7th edition of the AJCC cancer staging manual and the future of TNM. Ann Surg Oncol 2010;17:1471-4.

14. Gill IS, Kavoussi LR, Lane BR, Blute ML, Babineau D, Colombo JR Jr, et al. Comparison of 1,800 laparoscopic and open partial nephrectomies for single renal tumors. J Urol 2007;178:41-6.

15. Lane BR, Campbell SC, Gill IS. 10-year oncologic outcomes after laparoscopic and open partial nephrectomy. J Urol 2013; 190:44-9.

16. Springer C, Hoda MR, Fajkovic H, Pini G, Mohammed N, Fornara $\mathrm{P}$, et al. Laparoscopic vs open partial nephrectomy for T1 renal tumours: evaluation of long-term oncological and functional outcomes in 340 patients. BJU Int 2013;111:281-8.

17. Minervini A, Siena G, Antonelli A, Bianchi G, Bocciardi AM, Cosciani Cunico S, et al. Open versus laparoscopic partial nephrectomy for clinical T1a renal masses: a matched-pair comparison of 280 patients with TRIFECTA outcomes (RECORd Project). World J Urol 2014;32:257-63.

18. Reddy UD, Pillai R, Parker RA, Weston J, Burgess NA, Ho ET, et al. Prediction of complications after partial nephrectomy by RENAL nephrometry score. Ann R Coll Surg Engl 2014;96:475-9.

19. Kim JH, Park YH, Kim YJ, Kang SH, Byun SS, Kwak C, et al. Perioperative and long-term renal functional outcomes of robotic versus laparoscopic partial nephrectomy: a multicenter matched-pair comparison. World J Urol 2015;33:1579-84.

20. Bessede T, Bigot P, Bernhard JC, Pignot G, Boulière F, Verhoest $\mathrm{G}$, et al. Are warm ischemia and ischemia time still predictive factors of poor renal function after partial nephrectomy in the setting of elective indication? World J Urol 2015;33:11-5.

21. Ginzburg S, Uzzo R, Walton J, Miller C, Kurz D, Li T, et al. Residual parenchymal volume, not warm ischemia time, predicts ultimate renal functional outcomes in patients undergoing partial nephrectomy. Urology 2015;86:300-5. 